\title{
Age and birth cohort differences in the prevalence of common mental disorder in England: National Psychiatric Morbidity Surveys 1993-2007
}

Nicola Spiers, Paul Bebbington, Sally McManus, Traolach S. Brugha, Rachel Jenkins and Howard Meltzer

\section{Background}

There are concerns that the prevalence of mental disorder is increasing.

\section{Aims \\ To determine whether the prevalence of common adult mental disorders has increased over time, using age-period- cohort analysis. \\ Method \\ The study consisted of a pseudocohort analysis of a sequence of three cross-sectional surveys of the English household population. The main outcome was common mental disorder, indicated by a score of 12 or above on the Revised Clinical Interview Schedule (CIS-R). Secondary outcomes were neurotic symptoms likely to require treatment, indicated by a CIS-R score of 18 or over, and individual subscale scores for fatigue, sleep problems, irritability and worry.}

\section{Results}

There were 8670 participants in the 1993 survey, 6977 in the 2000 survey and 6815 in the 2007 survey. In men a significant increase in common mental disorder occurred between the cohort born in 1943-9 and that born in 1950-6 (odds ratio $1.4,95 \% \mathrm{Cl} 1.1-1.9$ ) but prevalence in subsequent cohorts remained largely stable. More extended increases in prevalence of sleep problems and mental disorders were observed in women, but not consistently across cohorts or measures.

\section{Conclusions}

We found little evidence that the prevalence of common mental disorder is increasing.

\section{Declaration of interest}

None.
Projections of future population mental health are important in planning treatment and services. However, such projections are currently restricted by the paucity of evidence about changes in population rates of mental disorder in the UK. ${ }^{1}$ There has been concern since the 1970s that the prevalence of mental disorders is increasing. ${ }^{2-4}$ General practice records have been used to estimate trends, but are subject both to time-related changes in diagnostic and prescribing behaviour, and to reporting issues. ${ }^{5}$ The British Adult Psychiatric Morbidity Survey (APMS) programme (http://www.mentalhealthsurveys.co.uk) included repeated, largescale cross-sectional surveys of the English adult population in the years 1993, 2000 and 2007, with standardised and essentially unchanged methods of evaluation. ${ }^{6-9}$ This programme was intended to monitor the mental health of people living in private households in England, in order to inform governmental objectives for mental health. ${ }^{10}$ Because the survey involved repeated cross-sectional surveys, it is possible to compare the health experience of successive birth cohorts, resampled as they aged over a period of 15 years. Although published APMS data indicate increases in the reporting of all neurotic symptoms in some age and gender groups, ${ }^{9}$ analysis by age and survey cannot provide a comprehensive view of the trends in health that emerge as cohorts age. For this study we used APMS data to describe age profiles and cohort differences, with the null hypothesis that successive birth cohorts experience the same prevalence of common mental disorder as they age.

\section{Method}

The methods used for the three national surveys are described in detail elsewhere. ${ }^{6-9}$ Adults living in private households in England were sampled using population-based multiphase probability sampling and interviewed in the first phase by lay interviewers. Although improvements were made in successive surveys, the emphasis was on using identical instruments wherever possible. Stratification of primary sampling units by region and socioeconomic characteristics was more fine-grained in 2007 than in 2000 and 1993, but in each case data were weighted to represent the English household population at the time of survey. The APMS sample size was designed to have the statistical power to estimate the prevalence of rare disorders $(0.5-1.0 \%)$ by age, gender and region, and therefore has sufficient power for an analysis of more common disorders by age, gender and birth cohort. Data on common mental disorders were available at all three points in time with identical questions. In 1993 and 2000 the Revised Clinical Interview Schedule (CIS-R) was an early section in the interview; $;^{12,13}$ in 2007 it came slightly later, after sections on health, caring, activities of daily living and medication. Fieldwork was carried out between April and September 1993, between March and September 2000, and between October 2006 and December 2007. Response rates for the household APMS were $79 \%$ in 1993 , declining to $69 \%$ in 2000 and $57 \%$ in 2007 . The paper-and-pencil questionnaires used in 1993 were replaced by computer-assisted interviewing in subsequent surveys; this is not thought to have had a substantial effect upon the results. ${ }^{11}$

Ethical approval was obtained for APMS 2000 and APMS 2007 from research ethics committees of the National Research Ethics Service appropriate for non-clinical populations.

\section{Measures}

Common mental disorder and neurotic symptoms were assessed using the CIS-R in all three surveys. ${ }^{12,13}$ Common mental disorder 
comprises depression and anxiety disorders, including generalised anxiety disorder, panic disorder, phobias and obsessive-compulsive disorder. The CIS-R is a structured schedule designed for lay interviewers. Questions refer to neurotic symptoms experienced in the past week or month. It provides a total score reflecting the overall severity of neurotic symptoms. In the analyses provided here, the total score was dichotomised at 12 and above, indicating that a common mental disorder was likely to be present, and at 18 and above, indicating a level of neurotic symptoms likely to require treatment. We also analysed the four most prevalent symptoms of those assessed by the CIS-R: sleep problems, fatigue, worry and irritability. These were regarded as present if the relevant symptom scored at least 2 from a total score of 4 , and contributed to the overall CIS-R score.

\section{Age-period-cohort analysis}

It is now accepted that age effects are more properly studied by observing birth cohorts as they age, rather than a set of rates by age at a given point in time. ${ }^{14}$ We need to distinguish between age, period and cohort effects. People may be exposed to risk of disorder because they are passing through an age of risk (an age effect). This will be apparent in differences in prevalence by age in all cohorts that have attained that age. Equally they may be exposed to disorder because they are passing through a time of risk. In this instance, different cohorts will experience the disorder at different ages, corresponding to their age at the relevant date (a period effect). Finally, birth cohorts may exhibit differing risk because of long-term effects on their health resulting from sharing a particular age and time of risk. For example, if child-rearing practices have a long-term effect on the child's mental health, trends in child-rearing practices would be expressed as a cohort difference in adults. A similar argument may be made for changes in social conditions during the transition to adulthood. ${ }^{15}$

It is difficult to separate age, period and cohort effects statistically, as this usually requires assumptions beyond those that the data allow. ${ }^{16}$ Our approach was to carry out an age-periodcohort analysis using a constraint-based approach, as described by Keyes et al. ${ }^{17}$ We conceptualised cohort differences in common mental disorder as arising from common influences on the experience of birth cohorts at key moments of development. Period effects on common mental disorder were conceptualised as contemporaneous influences that potentially confound the relationship between birth cohort and prevalence. In the logistic regression modelling, period effects were constrained to be zero, in order to estimate age and cohort effects. The validity of our constraint could not be tested empirically, but we examined its plausibility by reference to information from sources other than the APMS data-set, for example prevailing economic conditions indicated by changes in the rate of unemployment. In addition, we examined the effect on the results of choosing an alternative constraint.

\section{Participants}

Data were weighted to allow for survey design and differences in non-response by age, region and socioeconomic status, so that results are representative of the English household population of comparable age at the time of survey. ${ }^{9}$ The lower age limit was 16 years, and the upper age limit surveyed was 64 years in 1993 and 74 years in 2000, with no upper age limit in 2007. Because of the 7-year gaps between surveys, nine 7-year birth cohorts were defined, based on participants' ages at the time of the respective surveys. These covered birth dates from 1929 to 1991, and nine age groups from $16-22$ years through $72-78$ years. Birth cohort attribution is approximate, insofar as precise birth dates were not available. Those aged $72-74$ years in the 2000 survey were excluded because they did not form a complete 7-year birth cohort. Those aged 79 years and over when interviewed in 2007 were also excluded, as these cohorts were sampled only once and were strongly selected by survival, making interpretation difficult.

\section{Statistical analysis}

Data for men and women were analysed separately. The weighted prevalence of common mental disorder was graphed by age (the midpoint of the 7-year age group) and birth cohort. The svylogistic procedure in Stata version 11.0 for Windows was used to fit logistic regression models for age and birth cohort, taking into account the survey design. The final model was chosen using backwards selection to determine the adjustment for age, starting with cubic age. Using the age midpoint for each 7-year age group, the linear effect of age, together with indicator variables for 7-year birth cohort, were forced into the model. Thus, all models included a linear effect of age, and quadratic and cubic effects of age were included where statistically significant at the $5 \%$ level. Models were compared using the Wald test. ${ }^{18}$ Differences in prevalence of common mental disorder were estimated between successive pairs of birth cohorts. Smoothed profiles of disorder by age were plotted for the median cohort, born 1957 to 1963 .

\section{Results}

In 1993 a total of 8670 adults aged 16-64 years were interviewed in English private households; 6977 adults aged 16-71 years were interviewed in 2000, and 6815 adults aged 16-78 years were interviewed in 2007 (online Table DS1). Prevalence of common mental disorder and neurotic symptoms categorised by gender and survey are given in Table 1 . There was little change in rates for women, but prevalence of common mental disorder was somewhat higher in 2000 than in 1993 or 2007 for men. Missing items were minimal and did not affect the conclusions.

\section{Age and cohort differences}

The smoothed prevalence of common mental disorder (CIS-R score 12 or above) peaked between ages 40 years and 50 years in men, but did not change with age in women (Figs 1,2). The prevalence of symptoms likely to require treatment (CIS-R score 18 or above) also peaked after age 40 years in both men and women. The prevalence of irritability declined as cohorts aged, and the decline accelerated after age 40 years in men. In men, the prevalence of worry peaked around age 40 years, whereas the prevalence of worry declined with age in women. Fatigue increased with age in men, whereas in women prevalence was consistently high across the age groups, at around 36\%. Sleep problems increased markedly with age in both genders.

There was a step-change in male cohorts, with an increase in age-adjusted prevalence both of common mental disorder and of neurotic symptoms likely to require treatment. This occurred between the cohort born in 1943-9 and that born in 1950-6 (Table 2 and Fig. 3; online Table DS2). In part, these differences may have been driven by highly significant increases in fatigue and sleep problems. There was no further increase in prevalence across male cohorts born in 1950 and later, with the exception of a high prevalence of neurotic symptoms likely to require treatment in men aged 16-22 years in 2007, a cohort sampled only once.

As the cohort born in 1943-9 had lower rates than their successors on all measures at all three surveys, an age-period 


\begin{tabular}{|c|c|c|c|c|c|c|}
\hline & \multicolumn{6}{|c|}{ Survey } \\
\hline & \multicolumn{2}{|c|}{$\begin{array}{c}1993 \\
\text { Age } 16-64 \text { years }\end{array}$} & \multicolumn{2}{|c|}{$\begin{array}{c}2000 \\
\text { Age } 16-71 \text { years }\end{array}$} & \multicolumn{2}{|c|}{$\begin{array}{l}2007 \\
\text { Age } 16-78 \text { years }\end{array}$} \\
\hline & $n$ & $\%(95 \% \mathrm{Cl})$ & $n$ & $\%(95 \% \mathrm{Cl})$ & $n$ & $\%(95 \% \mathrm{Cl})$ \\
\hline \multicolumn{7}{|l|}{ Men } \\
\hline Common mental disorder (CIS-R score $\geqslant 12$ ) & 473 & $10.9(9.8-12.2)$ & 453 & $12.6(11.2-14.0)$ & 407 & $11.8(10.4-13.1)$ \\
\hline Symptoms likely to require treatment (CIS-R $\geqslant 18$ ) & 237 & $5.5(4.7-6.3)$ & 226 & $6.2(5.3-7.3)$ & 197 & $5.7(4.8-6.6)$ \\
\hline Fatigue & 884 & $20.6(19.1-22.0)$ & 828 & $23.0(21.2-24.7)$ & 773 & $22.4(20.5-24.2)$ \\
\hline Sleep problem & 897 & $20.8(19.2-22.5)$ & 857 & $23.8(21.9-25.6)$ & 797 & $23.1(21.4-24.8)$ \\
\hline Irritability & 814 & $18.9(17.3-20.5)$ & 676 & $18.8(17.0-20.5)$ & 514 & $14.9(13.5-16.2)$ \\
\hline Worry & 740 & $17.2(15.9-18.5)$ & 604 & $16.7(15.2-18.3)$ & 566 & $16.4(14.8-18.0)$ \\
\hline \multicolumn{7}{|l|}{ Women } \\
\hline Common mental disorder (CIS-R score $\geqslant 12$ ) & 782 & $18.1(16.6-19.6)$ & 671 & $18.5(16.9-20.1)$ & 671 & $18.9(17.4-20.4)$ \\
\hline Symptoms likely to require treatment $(C I S-R \geqslant 18)$ & 379 & $8.8(7.7-9.8)$ & 312 & $8.6(7.5-9.7)$ & 344 & $9.7(8.6-10.7)$ \\
\hline Fatigue & 1415 & $32.8(30.9-34.6)$ & 1172 & $32.4(30.4-34.4)$ & 1168 & $32.9(31.1-34.6)$ \\
\hline Sleep problem & 1226 & $28.4(26.7-30.1)$ & 1258 & $34.7(32.8-36.7)$ & 1302 & $36.7(34.9-38.4)$ \\
\hline Irritability & 1100 & 25.5 (23.9-27.0) & 815 & $22.5(20.9-24.1)$ & 742 & 20.9 (19.3-22.5) \\
\hline Worry & 988 & $22.9(21.3-24.4)$ & 791 & $21.8(20.0-23.6)$ & 779 & $21.9(20.4-23.5)$ \\
\hline \multicolumn{7}{|l|}{ Base $^{a}$} \\
\hline Men & 4300 & & 3606 & & 3454 & \\
\hline Women & 4318 & & 3622 & & 3553 & \\
\hline
\end{tabular}

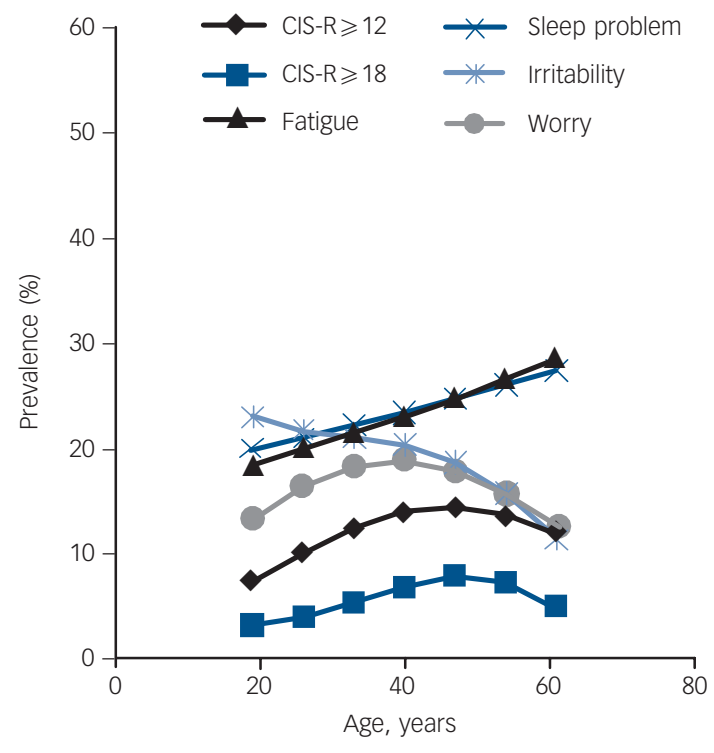

Fig. 1 Predicted age profiles of common mental disorder and neurotic symptoms from age-cohort models: men (CIS-R, Revised Clinical Interview Schedule).

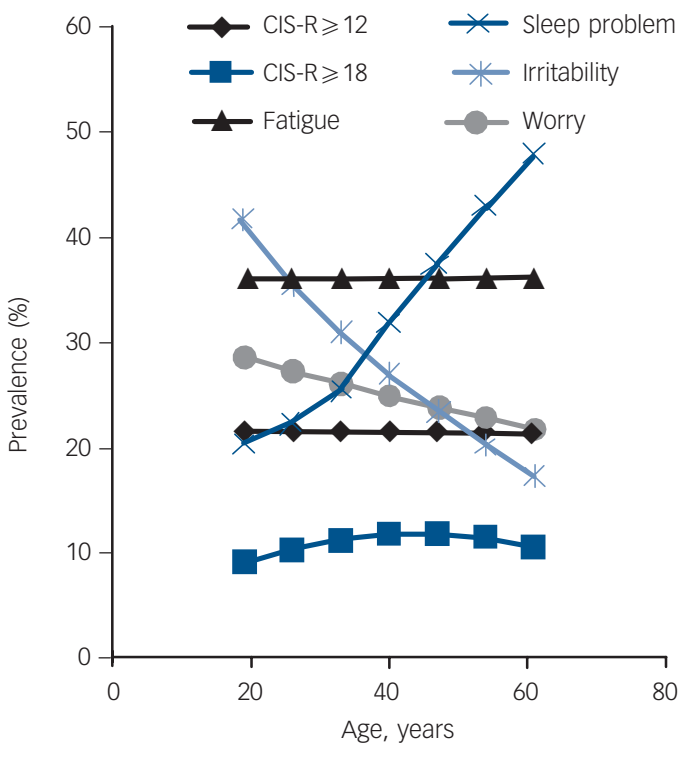

Fig. 2 Predicted age profiles of common mental disorder and neurotic symptoms from age-cohort models: women (CIS-R, Revised Clinical Interview Schedule). interaction seems unlikely. An alternative, given that the early cohorts were interviewed only in middle age, is to model the discontinuity purely in terms of age, with a rapid falling-off in prevalence with age after 50 years. However, the difference between the cohort born in 1943-9 and their successors remained statistically significant, even when additional parameters were included in the model to allow the functional form of the relationship with age to differ before and after age 43 years, fitting a more rapid decline in prevalence with age greater than 43 years.

There was an apparent peak in male prevalence of common mental disorder and neurotic symptoms in 2000, with a higher prevalence than in 1993 and 2007 across a number of cohorts and measures. Although this apparent period difference is ignored in our model, we carried out a sensitivity analysis by entering a term to allow for additional prevalence in 2000 only. This confirmed that the peak in 2000 does not confound our main finding of a discontinuity in the prevalence of common mental disorder between the male cohort born in 1950-6 and that born in 1943-9. Ignoring this period effect also leads to some overstatement of the trends in prevalence with age for both men and women in Figs 1 and 2, but the sensitivity analysis suggested that the impact of this was marginal.

Among women there were mixed trends in prevalence of common mental disorder across cohorts (Table 2, Fig. 4). Compared with the preceding cohort, those born in 1950-6 had a statistically greater prevalence of common neurotic disorder 


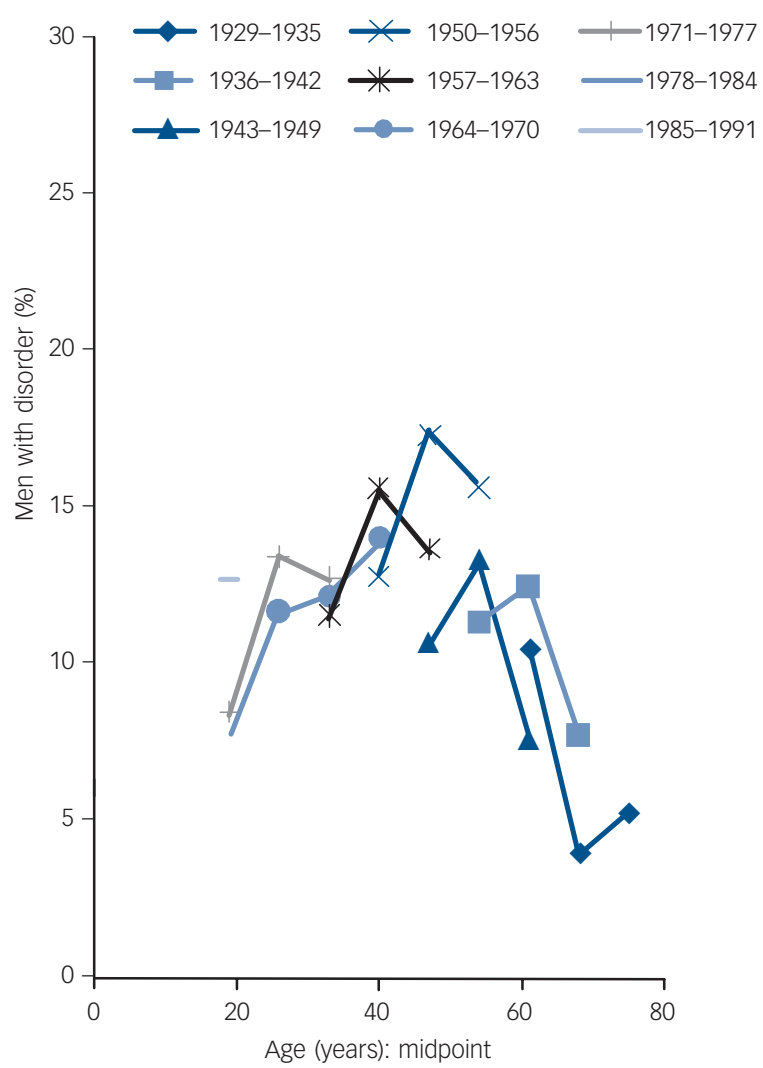

Fig. 3 Prevalence of common mental disorder (Revised Clinical Interview Schedule score $\geqslant 12$ ) in men, analysed by age and birth cohort.

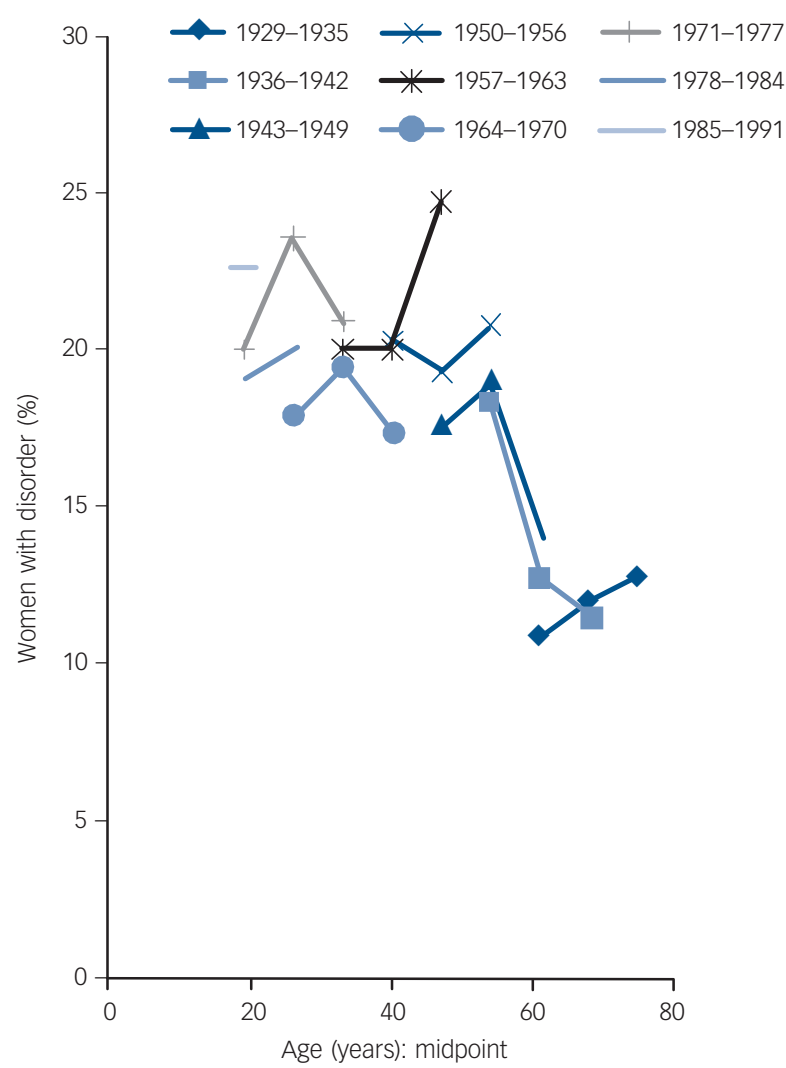

Fig. 4 Prevalence of common mental disorder (Revised Clinical Interview Schedule score $\geqslant 12$ ) in women, analysed by age and birth cohort. and of sleep problems and worry, but these differences between cohorts were less pronounced than in the male population. Women born between 1957 and 1963 had particularly high rates of common mental disorder and neurotic symptoms likely to require treatment when surveyed in 2007 aged $44-50$ years. The female prevalence of fatigue and irritability was high, up to $30 \%$ in some age groups, but remained stable or even decreased across succeeding cohorts. There were statistically significant increases in

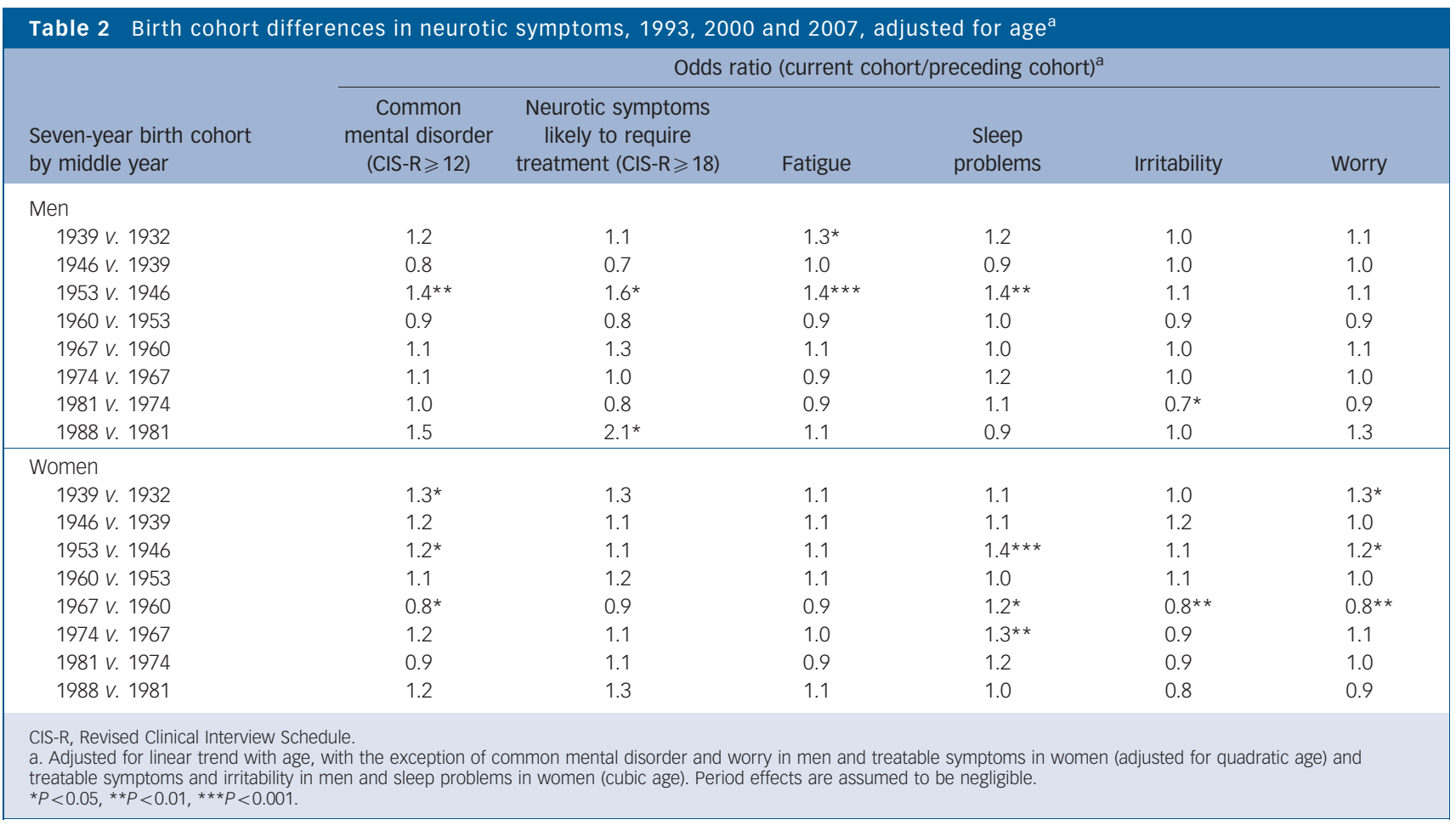


prevalence of sleep problems across four pairs of cohorts, and significant increases in prevalence of worry across two pairs of older cohorts. However, the prevalence of common mental disorder, irritability and worry declined significantly between the cohort born in 1957-63 and that born in 1964-70.

\section{Discussion}

This is the first analysis from the National Survey Programme to examine the mental health of successive English birth cohorts as they age. The main finding is of stability in prevalence of common mental disorder in men, with the exception of a step-change in prevalence between the cohort born in 1943-9 and their successors born in 1944-50. Those born in 1944-50 experienced a higher prevalence of disorder, which was then reproduced in subsequent birth cohorts. The pattern in Fig. 3, of lower rates in cohorts born before 1950 and then subsequent cohorts 'meshing' to form a stable prevalence by age profile, is also apparent for neurotic symptoms requiring treatment, fatigue and sleep problems in men.

There is greater uncertainty in the measures for women, with increased prevalence of sleep problems, but only limited evidence for an increase in other symptoms or common mental disorder in younger cohorts as they age.

\section{Comparison with other studies}

At one time it was common to analyse the age-specific incidence of mental disorders for successive cohorts of individuals (for example, Kessler et al). ${ }^{19}$ However, these studies used lifetime prevalence as a first indication of changes in risk in succeeding cohorts, which is subject to recall bias. Relatively small biases in recall can result in apparent cohort effects. ${ }^{20}$ Our study has the advantage of using point prevalence, so that such recall bias is not an issue, although - as with any health survey - there may be differential reporting due to the social unacceptability of admitting poor health.

Comparing self-reported mental health diagnoses in the Health Survey for England, Rice et al found a similar cohort difference to that reported here, with those born in 1950-4 having higher prevalence. ${ }^{21}$ An increase in prevalence of sleep loss through worry, attributed to the recession of the early 1990s, has been reported in the 1984 Health and Lifestyle survey, followed up in 1991-2. ${ }^{22}$ Given that unemployment started to rise in 1989 and peaked in 1993, a higher prevalence of neurotic symptoms might be expected in 1993 than in 2000 in the APMS. This is indeed the case for irritability and worry in women, but not for sleep problems and fatigue. However, the observed peak in prevalence in 2000 for men may indicate that prevalence peaked at a higher rate still after 1993 and was declining in 2000.

Data on trends in psychological distress from the 1958 National Child Development Study and the 1970 British Cohort Study have also been presented by Sacker \& Wiggins. ${ }^{23}$ Their finding of an increase in psychological distress up to age 40 years is consistent with our analyses. However, the reported increase in distress between the two cohorts in both genders contrasts with our findings for men, although the British Cohort Study only provided comparable data at ages 26 years and 30 years.

\section{Strengths and weaknesses}

Choosing to fit age-cohort models has the consequence that hypotheses about period differences cannot be tested. Further, it is possible that such influences confound the relationships that we have reported between birth cohort and disorder. To assess the potential for such confounding we have critically examined the possibility of period effects due to survey, treatment, and economic and social conditions.

A strength of this study is its use of standardised psychiatric evaluation with identical measures across three large, representative cross-sections of the English household population, spanning 15 years. Therefore, the possibility of spurious period differences arising artefactually from changes in survey methods is small. There is, however, a period difference in the response rate, which was clearly lower in 2007 than in 1993 and 2000. Although national surveys have the advantage of producing data on large representative samples, there is growing concern about falling response rates. ${ }^{24}$ The 2007 survey data used for this analysis were based on a response rate of $57 \%$. Nevertheless, great care was taken in trying to reduce biases by a sophisticated weighting procedure. In addition, recent non-response analyses of surveys from Scandinavian countries (especially those using a population register as a sampling frame) indicate very little non-response bias on a wide variety of physical and mental health measures. ${ }^{25-27}$

The potential impact of economic recession has already been discussed. Changes in treatment policy were also considered. There was a significant increase in the prescribing of antidepressants between 1993 and 2007, but relatively large numbers needed to treat and the fact that a clear majority of those with neurotic disorder do not seek treatment imply only a small effect on prevalence. $^{28}$ The proportions taking medication for a psychological or emotional problem (anxiolytics, hypnotics, antipsychotics or antidepressants) increased only slightly between 2000 and 2007 , from $5.5 \%$ (95\% CI $4.9-6.2$ ) to $5.8 \%$ (95\% CI 5.2-6.4). There was also an increase in those receiving counselling or therapy, from $1.5 \%$ (95\% CI 1.3-2.2) in 1993 to $2.3 \%$ (95\% CI $1.9-2.7)$ in 2000 and $2.7 \%$ (95\% CI 2.2-3.2) in 2007, but the numbers involved are small and unlikely to affect the estimates of age and cohort difference.

Although other solutions are possible, the age-cohort specification is a parsimonious and theoretically plausible account of the prevalence of common mental disorder in the male population. Indeed, it is a strength of our study that the results in men remained consistent, with only limited change in prevalence across analyses and outcomes. The pattern in women is less clear, and our conclusions about trends in women are tentative.

Some further potential limitations of the study must also be borne in mind. First, the outcomes presented here exclude some categories of mental disorder, including psychoses, severe affective disorders and substance misuse problems. Second, people who were homeless or living in institutions, likely to be older and in poorer mental health than those in private households, were not covered in these surveys, although they were surveyed elsewhere in the National Survey Programme. ${ }^{29,30}$ This subgroup is, however, quite small, at least at ages less than 65 years, and exclusions remained the same with each wave, so trends are unlikely to be affected.

\section{Implications}

Members of the cohort born in 1950-6, which was the first to experience higher rates of disorder in men, are forerunners of the 'baby boom' generation and were teenagers during the 1960s. This cohort was among the first in the UK to experience teenage culture, both home-grown and imported from the USA, and arguably the early impact of this on life choices may have been greater for men. The 1950-6 cohort may have experienced both wider opportunities and greater exposure to harmful substances when making the transition to adulthood than preceding cohorts. 
Our finding of subsequently stable rates contradicts popular media stories of a relentlessly rising tide of mental illness, ${ }^{31}$ at least for men. Stable prevalence in the male population, together with peaking of the prevalence of common mental disorder at about age 50 years, indicates that a large increase in projected rates of poor mental health is unlikely in the male population in the near future. Demand for treatment for common mental disorders may even fall as the male population ages, although the possibility of relatively short-term fluctuations due to economic conditions remains open and timely. ${ }^{32}$

Trends in common mental disorder in women are less clearly identified, with considerable increases in the prevalence of sleep problems, but no clear increase or even some decrease in other measures. Further research is needed to relate these age and cohort differences to drivers of mental health such as employment status and family composition.

Nicola Spiers, PhD, Department of Health Sciences, University of Leicester; Pau Bebbington, FRCPsych, Department of Mental Health Sciences, University College London; Sally McManus, MSc, National Centre for Social Research, London; Traolach S. Brugha, FRCPsych, Department of Health Sciences, University of Leicester; Rachel Jenkins, FRCPsych, Department of Epidemiology and International Mental Health Policy, Institute of Psychiatry, King's College, London; Howard Meltzer, PhD, Department of Health Sciences, University of Leicester, Leicester, UK

Correspondence: Nicola Spiers, Department of Health Sciences, University of Leicester, 22-28 Princess Road West, Leicester LE1 6TP, UK. Email: nas6@le.ac.uk

First received 2 July 2010, final revision 26 Oct 2010, accepted 20 Dec 2010

\section{Funding}

The Adult Psychiatric Morbidity Survey 2007 was commissioned by the National Health Service Information Centre for Health and Social Care, with funds from the Department of Health. The pseudocohort analysis was not funded.

\section{Acknowledgements}

The authors thank the participants and interviewers of the Adult Psychiatric Morbidity Survey, and Freya Tyrer and Nick Taub for helpful comments on this paper.

\section{References}

1 Foresight Mental Capital and Wellbeing Project. Final Project Report. UK Government Office for Science, 2008.

2 Klerman GL. The current age of youthful melancholia: evidence for increase in depression among adolescents and young adults. Br J Psychiatry 1988; 152: 4-14.

3 Murphy J. Trends in depression and anxiety: men and women. Acta Psychiat Scand 1986; 73: 113-27.

4 Compton WM, Conway KP, Stinson FS, Grant BF. Changes in the prevalence of major depression and comorbid substance use disorders in the United States between 1991-1992 and 2001-2002. Am J Psychiatry 2006; 163 2141-7

5 Rait G, Walters K, Griffin M, Buszewicz M, Petersen I, Nazareth I. Recent trends in the incidence of recorded depression in primary care. Br J Psychiatry 2009; 195: 520-4.

6 Meltzer H, Gill B, Petticrew M, Hinds K. OPCS Surveys of Psychiatric Morbidity in Great Britain, Report 1: The Prevalence of Psychiatric Morbidity Among Adults Living in Private Households. HMSO, 1995.

7 Meltzer $\mathrm{H}$, Jenkins R. The national survey of psychiatric morbidity in Great Britain. Soc Psychiatry Psychiatr Epidemiol 1995; 30: 1-4.
8 Singleton N, Bumpstead R, O'Brien M, Lee A, Meltzer H. Psychiatric Morbidity Among Adults Living In Private Households 2000. TSO (The Stationery Office), 2001.

9 McManus $\mathrm{S}$, Meltzer $\mathrm{H}$, Brugha $\mathrm{T}$, Bebbington $\mathrm{P}$, Jenkins R. Adult Psychiatric Morbidity in England, 2007: Results of a Household Survey. National Health Service Information Centre for Health and Social Care, 2009.

10 Jenkins $\mathrm{R}$, Meltzer $\mathrm{H}$, Bebbington $\mathrm{P}$, Brugha $\mathrm{T}$, Farrell $\mathrm{M}$, McManus $\mathrm{S}$, et al. The British Mental Health Survey Programme: achievements and latest findings. Soc Psychiatry Psychiatr Epidemiol 2009; 44: 899-904.

11 Baker RP, Bradburn NM, Johnson RA. Computer-assisted personal interviewing: an experimental evaluation of data quality and cost. I Off Stat 1995; 11: 413-31.

12 Lewis G, Pelosi AJ, Araya A, Dunn G. Measuring psychiatric disorder in the community: a standard assessment for use by lay interviewers. Psychol Med 1992; 22: 465-86.

13 Das-Munshi J, Goldberg D, Bebbington PE, Bhugra DK, Brugha TS, Dewey ME, et al. Public health significance of mixed anxiety and depression: beyond current classification. Br J Psychiatry 2008; 192: 171-7.

14 Smith HL. Advances in age-period-cohort analysis. Sociol Methods Res 2008; 36: 287-96.

15 Sacker A, Cable N. Transitions to adulthood and psychological distress in young adults born 12 years apart: constraints on and resources for development. Psychol Med 2010; 40: 301-13.

16 Klerman GL, Weismann MM. Increasing rates of depression. JAMA 1989; 261: 2229-35.

17 Keyes, KM, Utz RL, Robinson W, Li G. What is a cohort effect? Comparison of three statistical methods for modeling cohort effects in obesity prevalence in the United States, 1971-2006. Soc Sci Med 2010; 70: 1100-8.

18 Armitage P, Berry G, Matthews JNS. Statistical Methods in Medical Research (4th edn). Blackwell, 2002

19 Kessler RC, McGonagle KA, Nelson CB, Hughes M, Swartz M, Blazer DG Sex and depression in the National Comorbidity Survey. II: Cohort effects. J Affect Disord 1994; 30: 15-26.

20 Giuffra LA, Risch N. Diminished recall and the cohort effect of major depression: a simulation study. Psychol Med 1994; 24: 375-83.

21 Rice NE, Lang IA, Henley W, Melzer D. Baby boomers nearing retirement: the healthiest generation? Rejuvenation Res 2010; 13: 105.

22 Dregan A, Armstrong D. Age, cohort and period effects in the prevalence of sleep disturbances among older people: the impact of economic downturn. Soc Sci Med 2009; 69: 1432-8.

23 Sacker A, Wiggins RD. Age-period-cohort effects on inequalities in psychological distress, 1981-2000. Psychol Med 2002; 32: 977-90.

24 De Leeuw $\mathrm{E}$, de Heer $\mathrm{W}$. Trends in household survey nonresponse: a longitudinal and international comparison. In Survey Nonresponse (eds RM Groves, DA Dillman, JL Elting, RJA Little): 41-54. Wiley, 2002.

25 De Winter AF, Oldehinkel AJ, Veenstra R, Brunnekreef JA, Verhulst FC, Ormel $J$. Evaluation of non-response bias in mental health determinants and outcomes in a large sample of pre-adolescents. Eur J Epidemiol 2005; 20 : $173-81$

26 Korkeila K, Suominen S, Ahvenainen J, Ojanlatva A, Rautava P, Helenius $\mathrm{H}$ et al. Non-response and related factors in a nation-wide health survey. Eur J Epidemiol 2001; 17: 983-1057.

27 Søgaard AJ, Selmer R, Bjertness E, Thelle D. The Oslo Health Study: the impact of self-selection in a large, population-based survey. Int J Equity Health 2004; 3: 3 .

28 Brugha TS, Bebbington PE, Singleton N, Melzer D, Jenkins R, Lewis G, et al. Trends in service use and treatment for mental disorders in adults throughout Great Britain. Br J Psychiatry 2004; 185: 378-84.

29 Singleton N, Meltzer H, Gatward R, Coid J, Deasy D. Psychiatric Morbidity Among Prisoners in England and Wales. TSO (The Stationery Office), 1998.

30 Gill B, Meltzer H, Hinds K, Petticrew M. OPCS Surveys of Psychiatric Morbidity in Great Britain, Report 7: Psychiatric Morbidity Among Homeless People. HMSO, 1996.

31 Hope J. The anxious generation: $7 m$ Britons racked with worry over recession, crime, health....and Big Brother state. Mail Online, 14 April 2009

32 Dorling D. Unemployment and health. BMJ 2009; 338: b829. 